\title{
Investigation of structural mechanisms of laser-written waveguide formation through third-harmonic microscopy
}

\author{
Jun Guan, ${ }^{1,3}$ Xiang Liu, ${ }^{1}$ and Martin J. Booth ${ }^{1,2, *}$ \\ ${ }^{1}$ Department of Engineering Science, University of Oxford, Parks Road, Oxford OX1 3PJ, UK \\ ${ }^{2}$ Centre for Neural Circuits and Behaviour, University of Oxford, Mansfield Road, Oxford OX1 3SR, UK \\ ${ }^{3}$ e-mail: jun.guan@eng.ox.ac.uk \\ ${ }^{*}$ Corresponding author: martin.booth@eng.ox.ac.uk
}

Received 13 November 2018; revised 4 December 2018; accepted 4 December 2018; posted 5 December 2018 (Doc. ID 351841); published 15 February 2019

\begin{abstract}
The mechanisms of laser-induced modification of transparent materials are complex combinations of different processes that depend on the material itself and a range of processing parameters. As such, the mechanisms are still subject to ongoing study. We use a custom-built adaptive third-harmonic generation (THG) microscope to study those mechanisms. New femtosecond-laser-written phenomena are revealed through this method of imaging. This Letter, together with previous reports by Miyamoto Opt. Express 24, 25718 (2016) and Fernandez J. Phys. D 48,155101 (2015) suggest that the distribution of the generated plasma during writing is responsible for the newly revealed phenomena.
\end{abstract}

Published by The Optical Society under the terms of the Creative Commons Attribution 4.0 License. Further distribution of this work must maintain attribution to the author(s) and the published article's title, journal citation, and DOI.

https://doi.org/10.1364/OL.44.001039

By virtue of its rapid prototyping nature and compatibility with different materials, femtosecond-laser direct writing has become a popular fabrication technology for photonic integrated circuits (PICs) for photon-based quantum information processing (QIP) [1-8] among other application areas [9-17]. In particular, its intrinsic three-dimensional (3D) fabrication capability uniquely enables the fabrication of PICs involving complex 3D photonic structures [18-21]. As in other application areas, the development of QIP demands up-scaling and much better performance of the PIC at the same time. To meet those demands, as well as in the interest of fundamental study, we need to understand the mechanisms of laser-induced material modification which have been extensively studied over the last two decades $[10,13,22-24]$. The exact mechanisms of laser-induced material modification are still subject to ongoing study $[13,23,24]$; this is due to not only the complex natures of the mechanisms, but also the limited ways we have to study them, particularly when considering the fine cross-sectional structure of the waveguides. Here, based on a custom-built adaptive 3D third-harmonic generation (THG) microscope, we demonstrate a new way to study those mechanisms.

Waveguides were fabricated with the second harmonic of a regenerative amplified $\mathrm{Yb}: \mathrm{KGW}$ laser (170 fs pulse duration, $1 \mathrm{MHz}$ repetition rate, $514 \mathrm{~nm}$ wavelength). The power of the circularly polarized laser beam was regulated by a rotating half-wave plate and a polarizing beam splitter before being focused inside a glass chip with a 0.5 NA objective lens. The glass chip was transversely scanned relative to the focus to inscribe the integrated photonic components [25]. A custom-built adaptive 3D THG microscope was used to image the waveguides (full details are in Ref. [26]). Briefly, this consisted of a Cr:forsterite laser (pulse duration $65 \mathrm{fs}$, repetition rate $100 \mathrm{MHz}$, wavelength of $1235 \mathrm{~nm}$ ), a two-axis galvanometer scanner, a deformable membrane mirror for aberration correction, a $1.15 \mathrm{NA}$ water immersion objective lens, and a piezo $z$-translation stage. THG emission was collected in the forward direction. During THG imaging, the pixel dwell times ranged from 0.5 to $8 \mu$ s. Aberrations were corrected by sequentially adjusting the amplitudes of the Zernike polynomial modes added to the deformable mirror in order to maximize the total image intensity. First, system aberrations were corrected by optimizing the THG signal at the surface of a waveguide chip. Then the remaining aberrations, which were introduced by the refractive index mismatch between the immersion medium and the chip substrate at the imaging depth, were corrected [26]. We note here that while the THG depends on the third-order nonlinear optical properties of the material, among other factors, in practice, the measured THG signal correlates spatially to changes in refractive-index profiles $[27,28]$ such that a rapid spatial transition between different refractive indices typically correlates with a high signal. In the following, unless otherwise noted, all the waveguide cross-sectional profiles are THG profiles.

For demonstrations, waveguides were written inside a borosilicate glass (Corning EAGLE 2000) chip with different scan speeds and pulse energies. Images of their cross sections were 
taken with the THG microscope, as shown in Fig. 1, from which we can see how the waveguide cross-sectional THG profiles, along with writing parameters, evolved. First of all, under the same pulse energy, the cross-sectional profile became more complex with decreasing scan speeds, which indicated more complex refractive index changes across the section at lower scan speeds. This is contradictory to the purely thermaleffect-based theory of photonic structure formation with highrepetition-rate femtosecond lasers [29], since a decrease of the scan speed under constant pulse energy means an increase of the thermal effect which, according to that theory, should have resulted in smoother refractive index changes and a simpler THG profile. Secondly, under the same scan speed, as pulse energy was increased, areas of stronger THG signals shifted from the top to the bottom of the cross-sectional profile. The detailed evolution of the THG profile along pulse energy was more complex.

Another striking phenomenon was the sudden emergence of a much stronger THG signal at the bottom of THG profiles 4-7, 11-14, 20-21, 27-28, and 35 in Fig. 1. As exemplified in Fig. 2(a), these areas corresponded to the dark spots at the bottom of the corresponding conventional transmission microscopy images, which were aligned with the focus of the objective lens [29]; the transmission microscopy images were taken with a Zeiss Axioplan 2 microscope. Although there was no obvious difference between those dark spots on

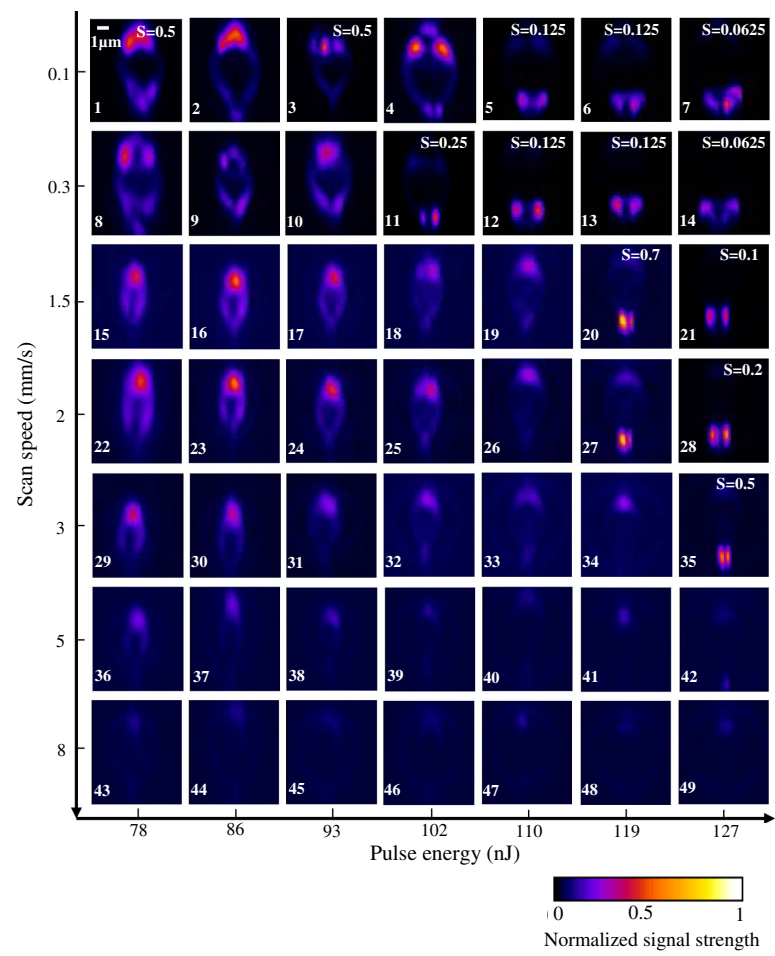

Fig. 1. Cross-sectional THG images (in false color) of waveguides written in Corning Eagle 2000 glass with different pulse energies and scan speeds; the number at the bottom left corner of a THG image is the waveguide serial number; $S$ is the normalized integration time of the THG microscope detector, which is marked on the top right corner of THG images when it is set below 1 to avoid image saturation; some of the THG images were scaled slightly differently to aid visualization. The color map (color map 1) is chosen to highlight THG signal contrast. (a)

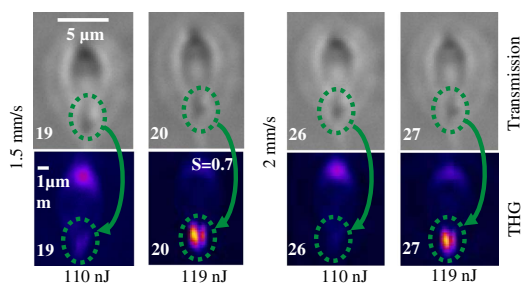

(b)

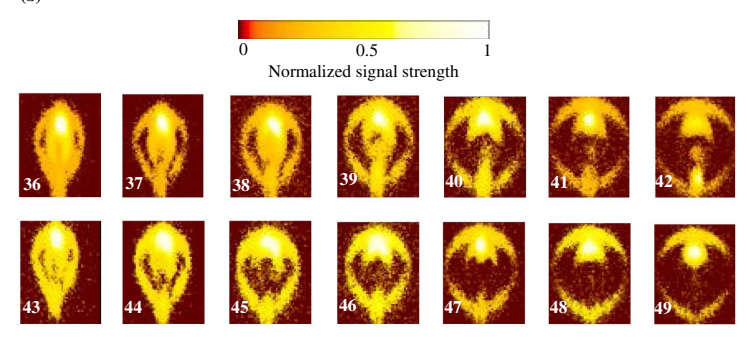

Fig. 2. (a) Comparison between transmission microscopy images and their corresponding THG images for waveguides 19-20 and 26-27. (b) THG images of waveguide 36-49 are rendered in another color map (color map 2) to highlight the waveguide profiles.

transmission microscopy images of waveguides 19-20 and 26-27, the contrasts in terms of THG signal strength on their THG images at corresponding locations were striking. This suggests that there were actually two types of a low-density region: a type I low-density region, which was formed through non-explosive rarefaction, for instance, the marked regions on images of waveguides 19 and 26 in Fig. 2(a); and a type II lowdensity region represented by the marked regions on the images of waveguides 20 and 27 , which is broadly recognized to be formed by micro-explosion $[23,30]$. The slight changes of writing pulse energy from waveguides 19 and 26 to waveguides 20 and 27 , respectively, implies that there was a threshold-like sharp transition between the formations of the two types of low-density regions. This corroborates the micro-explosion theory $[23,30]$. Our new observations allow us to enhance this theory with the revelation of a type I low-density region. We also demonstrate how to identify the two types of low-density regions and determine the transition threshold between them, using the THG microscope images.

The adaptive THG microscope also enabled us to study the micro-morphology of a waveguide from structural and dimensional aspects. To reveal the structural and geometrical profiles of the written waveguides, the THG images shown in Fig. 1 were re-rendered with a different color map, as exemplified in Fig. 2(b). The structural evolution of the waveguides is summarized in Fig. 3(a), from which we can see that the waveguides written with moderate scan speeds and pulse energies clearly exhibited so called "core-cladding" structures [29]. The cladding gradually disappeared as the scan speed and pulse energy were decreased, and the core gradually disappeared with an increasing scan speed and pulse energy. Type II low-density regions emerged at high pulse energies and low-to-moderate scan speeds.

This imaging evidence shows that the well-accepted material modification theory based on thermal effects using highrepetition-rate femtosecond lasers [13,23,29] does not fully 

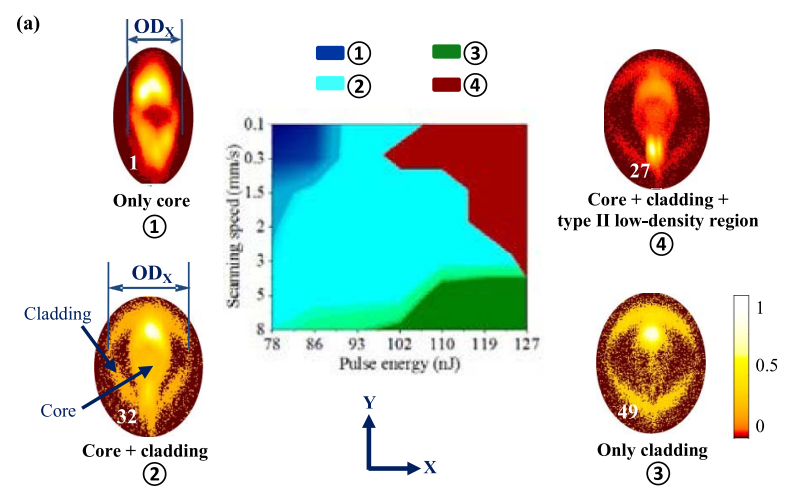

(b)

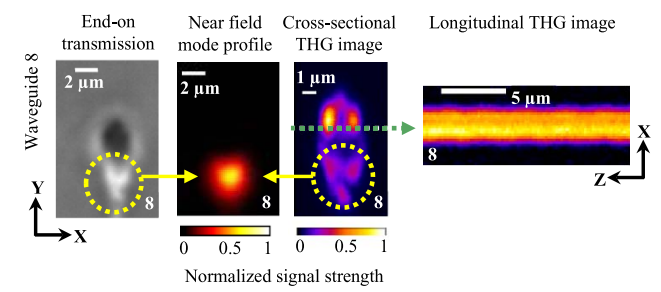

Fig. 3. (a) Structural evolution of waveguides shown in Fig. 1. Along the variation of writing parameters, the waveguide cross sections can exhibit four types of structures, as exemplified by the four waveguide THG images; each structure type is represented by a color in the evolution graph; here the THG images were re-rendered with color map 2 to reveal structural details. (b) End-on transmission image, near-field mode profile (in false color), and cross-sectional and longitudinal THG images of waveguide 8 . The region marked with dashed yellow circles is a light-guiding region; the green arrow is used to indicate the position where a longitudinal THG image was taken.

explain the observed phenomena. For example, the waveguides written with a low scan speed exhibit more complex THG cross-sectional profiles than those written with a high scan speed, but the same pulse energy. Another well-investigated theory based on ion migration $[31,32,23]$ cannot fully explain the phenomena of waveguide structure evolution shown in Fig. 3(a). It is apparent that even the combination of all the existing theories $[13,23,29,31,32]$ is still insufficient for the explanation of the revealed phenomena. In the following, building on prior works $[31,33]$, we show that the generated plasma during laser writing plays an important role for the explanation of those observations.

Waveguide 8 is taken as an example to directly compare conventional transmission end-on images and corresponding cross-sectional THG images, as shown in Fig. 3(b), from which we can see that the THG microscope resolves the waveguide structure in more detail, bearing in mind that THG is correlated to a refractive index change instead of the refractive index itself. In Fig. 3(b), the guiding region of waveguide 8 is marked with the yellow dashed circle. The near-field mode profile was recorded with $788 \mathrm{~nm}$ laser excitation. From the longitudinal THG image of waveguide 8 , which was taken at the position indicated on the cross-sectional THG image in Fig. 3(b), we can see the structure is invariant along the waveguide.

The waveguide dimensions were measured from the THG images. The pixel size has been calibrated through features with known dimensions. The waveguide outer dimension along the $X$ direction $\left(O D_{X}\right)$ is defined as the size of cladding the in the $O D_{X}$ when there is a cladding structure or size of the core for
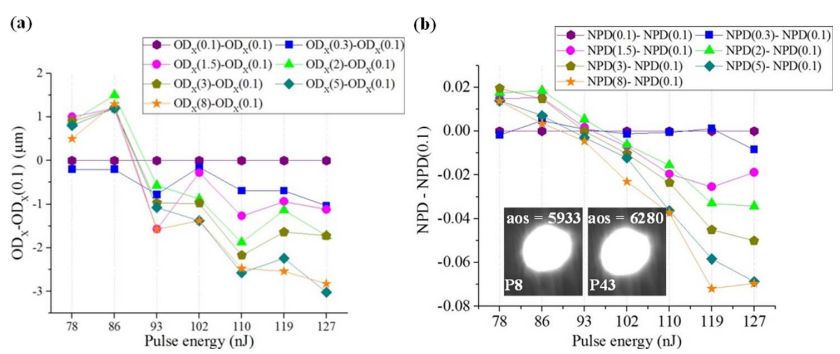

Fig. 4. (a) Difference between the outer dimensions in $X O D_{X}$ of all waveguides in Fig. 1 and those of waveguides written with a scan speed of $0.1 \mathrm{~mm} / \mathrm{s}\left(O D_{X}(0.1)\right)$; in the legends of (a) and (b), the numbers in parentheses are scan speeds in $\mathrm{mm} / \mathrm{s}$. (b) Difference between NPD and NPD(0.1); NPD is the normalized dimension of the plasma emission spot generated during waveguide writing; $\mathrm{NPD}(0.1)$ is the $\mathrm{NPD}$ at a scan speed of $0.1 \mathrm{~mm} / \mathrm{s}$; two recorded plasma emission spots $\mathrm{P} 8$ and $\mathrm{P} 43$ are shown in the inset as examples, which were recorded during writing waveguides 8 and 43 , respectively.

waveguide without a cladding structure, as shown in structure (2) and (1), respectively, in Fig. 3(a). The differences between the waveguide outer dimensions in $\mathrm{X}$ direction $O D_{X}$ and those of waveguides written with a scan speed of $0.1 \mathrm{~mm} / \mathrm{s}\left(O D_{X}(0.1)\right)$ are presented in Fig. 4(a). This reveals another counterintuitive phenomenon: the waveguides written with a pulse energy of 78 and $86 \mathrm{~nJ}$ and a scan speed of 0.1 and $0.3 \mathrm{~mm} / \mathrm{s}$, which have a core-only structure at the top left corner in the middle graph of Fig. 3(a), exhibit smaller $O D_{X}$ than those written with a higher scan speed. This is opposite the well-accepted thermal theory $[13,23,29]$, according to which the waveguides written with a lower scan speed should exhibit greater $O D_{X}$ than those written with the same pulse energy, but a higher scan speed. This is also inexplicable with other existing theories [23,31,32]. However, we found out that this phenomenon correlated with the dimensional evolution of the plasma emission spot generated during waveguide writing in the same dimension.

During waveguide writing, the generated plasma emission spots were recorded through the writing objective lens with an in-line camera (Baumer VLU-12M). Due to the large dynamic range of plasma intensities and background light resulting from varied writing parameters during these experiments, we chose to operate the CCD camera in a saturated regime. The camera settings, which were chosen to guarantee that there was always saturation only in the plasma emission spot, as exemplified in the inset of Fig. 4(b), were fixed throughout writing of all waveguides. We found that the repeatability of this experiment over the large dynamic range could only be achieved with this saturation-based method; a longer CCD integration time, which results in saturation, helped reduce the influence of noises. The validity and measurement repeatability of our saturation approach can be verified through a simple test such as measuring the dimension of the near-field profile of a laser beam with saturation and non-saturation settings under different background lighting conditions. The dimension of the plasma emission spot in the $X Z$ plane was decided based on the area corresponding emission intensity above certain intensity $E I_{d}$, bearing in mind that we expect the threshold of refractive index modification to be related to plasma intensity. For repeatable measurements, $E I_{d}$ was chosen to be the camera saturation intensity. To remove the influence of the definition 
itself, the dimensions of plasma emissions were normalized. The normalized plasma dimension (NPD) was defined as $\mathrm{NPD}=\sqrt{\mathrm{aos}} / \sqrt{\mathrm{aos}_{\max }}$, where aos is the area of saturation region of the recorded plasma emission spot, which is represented by the number of saturated CCD pixels. In addition, aos $\max _{\max }$ is the maximum value among all aos. It is worth mentioning that all the waveguides were written in the same depth.

Similar to the presentation in Fig. 4(a), in Fig. 4(b), the normalized plasma dimension is presented in the form of (NPD)$\operatorname{NPD}(0.1)$, where $\operatorname{NPD}(0.1)$ is the NPD at a scan speed of $0.1 \mathrm{~mm} / \mathrm{s}$. From Figs. 4(a) and 4(b), we can see that the same trends can be observed between the outer dimensions of written structures in a transverse $O D_{X}$ and those of the emission plasma spots generated during writing. Based on this correlation, together with a previous report on plasma dynamics [33] and controlling plasma distribution [31] during writing, we believe that the spatiotemporal distribution of the generated plasma during writing is responsible for the aforementioned unexplained phenomena. As discovered in Ref. [33], the ascending speed of the tandem plasmas is slowed down gradually when they approach the top; this could be the reason for the stronger refractive index change at the top of each waveguide shown in Fig. 1 of this Letter, since a stronger or longer interaction between the plasma and material could result in a stronger refractive index change. We speculate that the slowed down temporal and plasma spatial distributions would be different when the writing parameters change, so they could be reasons for the THG profile evolution, along with the writing parameters in Fig. 1. The sudden emergence and quick diffusion of the small elongated plasma at the bottom (geometrical focus) of frame numbers 2 and 31 of the second figure in Ref. [33] also appear to be well aligned with the formation of the two types of low-density regions, assuming that the intensity of that small plasma would increase with an increase of pulse energy. The correlation between the spatial distribution of the plasma and that of the waveguide refractive index change is demonstrated in Ref. [31] and, in this Letter, from different aspects. It is worth noting that the periodic movement of plasma shown in Ref. [33] might lead to a periodic variation of a waveguide cross-sectional profile along the waveguide, but we have not yet seen this variation. We speculate that this could be because the period of the plasma movement would change as the scan speed changes, which makes direct observation of the periodic variation along the waveguide very hard, despite the sub-micrometer lateral resolution of the THG microscope.

To summarize, based on the ability of the THG microscope to reveal structure in $3 \mathrm{D}$ and on the sub-micrometer scale, our method enables us to see laser writing phenomena that are not otherwise visible using conventional imaging methods. The method provides us a new pathway toward better understanding of laser-induced modification. Existing theories of laser material interaction in waveguide formation do not fully explain the revealed phenomena. The observed correlation between the waveguide dimension and that of the plasma emission generated during writing, together with previous reports, suggest that the spatiotemporal distribution of the plasma during writing is a key to the explanation of those newly revealed phenomena.

Funding. Engineering and Physical Sciences Research Council (EPSRC) (EP/K034480/1, EP/M013243/1).

\section{REFERENCES}

1. L. Sansoni, F. Sciarrino, G. Vallone, P. Mataloni, A. Crespi, R. Ramponi, and R. Osellame, Phys. Rev. Lett. 105, 200503 (2010).

2. A. Crespi, R. Ramponi, R. Osellame, L. Sansoni, I. Bongioanni, F. Sciarrino, G. Vallone, and P. Mataloni, Nat. Commun. 2, 566 (2011).

3. A. Crespi, R. Osellame, R. Ramponi, V. Giovannetti, R. Fazio, L. Sansoni, F. De Nicola, F. Sciarrino, and P. Mataloni, Nat. Photonics 7, 322 (2013).

4. G. Di Giuseppe, L. Martin, A. Perez-Leija, R. Keil, F. Dreisow, S. Nolte, A. Szameit, A. F. Abouraddy, D. N. Christodoulides, and B. E. A. Saleh, Phys. Rev. Lett. 110, 150503 (2013).

5. M. Tillmann, B. Dakić, R. Heilmann, S. Nolte, A. Szameit, and $P$. Walther, Nat. Photonics 7, 540 (2013).

6. Z. Chaboyer, T. Meany, L. G. Helt, M. J. Withford, and M. J. Steel, Sci. Rep. 5, 9601 (2015).

7. T. Meany, D. N. Biggerstaff, M. A. Broome, A. Fedrizzi, M. Delanty, M. J. Steel, A. Gilchrist, G. D. Marshall, A. G. White, and M. J. Withford, Sci. Rep. 6, 25126 (2016).

8. M. A. Ciampini, A. Orieux, S. Paesani, F. Sciarrino, G. Corrielli, A. Crespi, R. Ramponi, R. Osellame, and P. Mataloni, Light Sci. Appl. 5, e16064 (2016).

9. Y. Cheng, K. Sugioka, and K. Midorikawa, Opt. Lett. 29, 2007 (2004).

10. R. R. Gattass and E. Mazur, Nat. Photonics 2, 219 (2008).

11. M. Ams, G. D. Marshall, P. Dekker, J. A. Piper, and M. J. Withford, Laser Photonics Rev. 3, 535 (2009).

12. R. R. Thomson, T. A. Birks, S. G. Leon-Saval, A. K. Kar, and J. Bland-Hawthorn, Opt. Express 19, 5698 (2011)

13. R. Osellame, G. Cerullo, and R. Ramponi, Femtosecond Laser Micromachining Photonic and Microfluidic Devices in Transparent Materials (Springer, 2012).

14. B. Sun, P. S. Salter, and M. J. Booth, Appl. Phys. Lett. 105, 231105 (2014).

15. G. Douglass, F. Dreisow, S. Gross, S. Nolte, and M. Withford, Opt. Express 23, 21392 (2015).

16. W. Nie, R. Li, C. Cheng, Y. Chen, Q. Lu, C. Romero, J. R. Vázquez de Aldana, X. Hao, and F. Chen, Sci. Rep. 7, 46162 (2017).

17. N. Buch-Månson, A. Spangenberg, L. P. C. Gomez, J.-P. Malval, O. Soppera, and K. L. Martinez, Sci. Rep. 7, 9247 (2017).

18. M. C. Rechtsman, J. M. Zeuner, Y. Plotnik, Y. Lumer, D. Podolsky, F. Dreisow, S. Nolte, M. Segev, and A. Szameit, Nature 496, 196 (2013).

19. N. Spagnolo, C. Vitelli, L. Aparo, P. Mataloni, F. Sciarrino, A. Crespi, R. Ramponi, and R. Osellame, Nat. Commun. 4, 1606 (2013).

20. L. Sansoni, F. Sciarrino, G. Vallone, P. Mataloni, A. Crespi, R. Ramponi, and R. Osellame, Phys. Rev. Lett. 108, 010502 (2012).

21. K. Poulios, R. Keil, D. Fry, J. D. A. Meinecke, J. C. F. Matthews, A. Politi, M. Lobino, M. Gräfe, M. Heinrich, S. Nolte, A. Szameit, and J. L. O'Brien, Phys. Rev. Lett. 112, 143604 (2014).

22. K. Sugioka and Y. Cheng, Appl. Phys. Rev. 1, 041303 (2014).

23. D. Tan, K. N. Sharafudeen, Y. Yue, and J. Qiu, Prog. Mater. Sci. 76, 154 (2016).

24. A. Fuerbach, S. Gross, D. Little, A. Arriola, M. Ams, P. Dekker, and M. Withford, Proc. SPIE 9983, 99830W (2016).

25. J. Guan, X. Liu, P. S. Salter, and M. J. Booth, Opt. Express 25, 4845 (2017).

26. A. Jesacher, A. Thayil, K. Grieve, D. Débarre, T. Watanabe, T. Wilson, S. Srinivas, and M. Booth, Opt. Lett. 34, 3154 (2009).

27. M. Müller, J. Squier, K. R. Wilson, and G. J. Brakenhoff, J. Microsc. 191, 266 (1998)

28. G. D. Marshall, A. Jesacher, A. Thayil, M. J. Withford, and M. Booth, Opt. Lett. 36, 695 (2011)

29. S. M. Eaton, H. Zhang, M. L. Ng, J. Li, W.-J. Chen, S. Ho, and P. R. Herman, Opt. Express 16, 9443 (2008).

30. S. Kanehira, J. Si, J. Qiu, K. Fujita, and K. Hirao, Nano Lett. 5, 1591 (2005).

31. T. T. Fernandez, J. Siegel, J. Hoyo, B. Sotillo, P. Fernandez, and J. Solis, J. Phys. D 48, 155101 (2015).

32. T. T. Fernandez, M. Sakakura, S. M. Eaton, B. Sotillo, J. Siegel, J. Solis, Y. Shimotsuma, and K. Miura, Prog. Mater. Sci. 94, 68 (2018).

33. I. Miyamoto, Y. Okamoto, R. Tanabe, Y. Ito, K. Cvecek, and M. Schmidt, Opt. Express 24, 25718 (2016). 